DOI: $10.18287 / 2409-4579-2018-4-4-23-26$

УДК 535.374:621.375.8

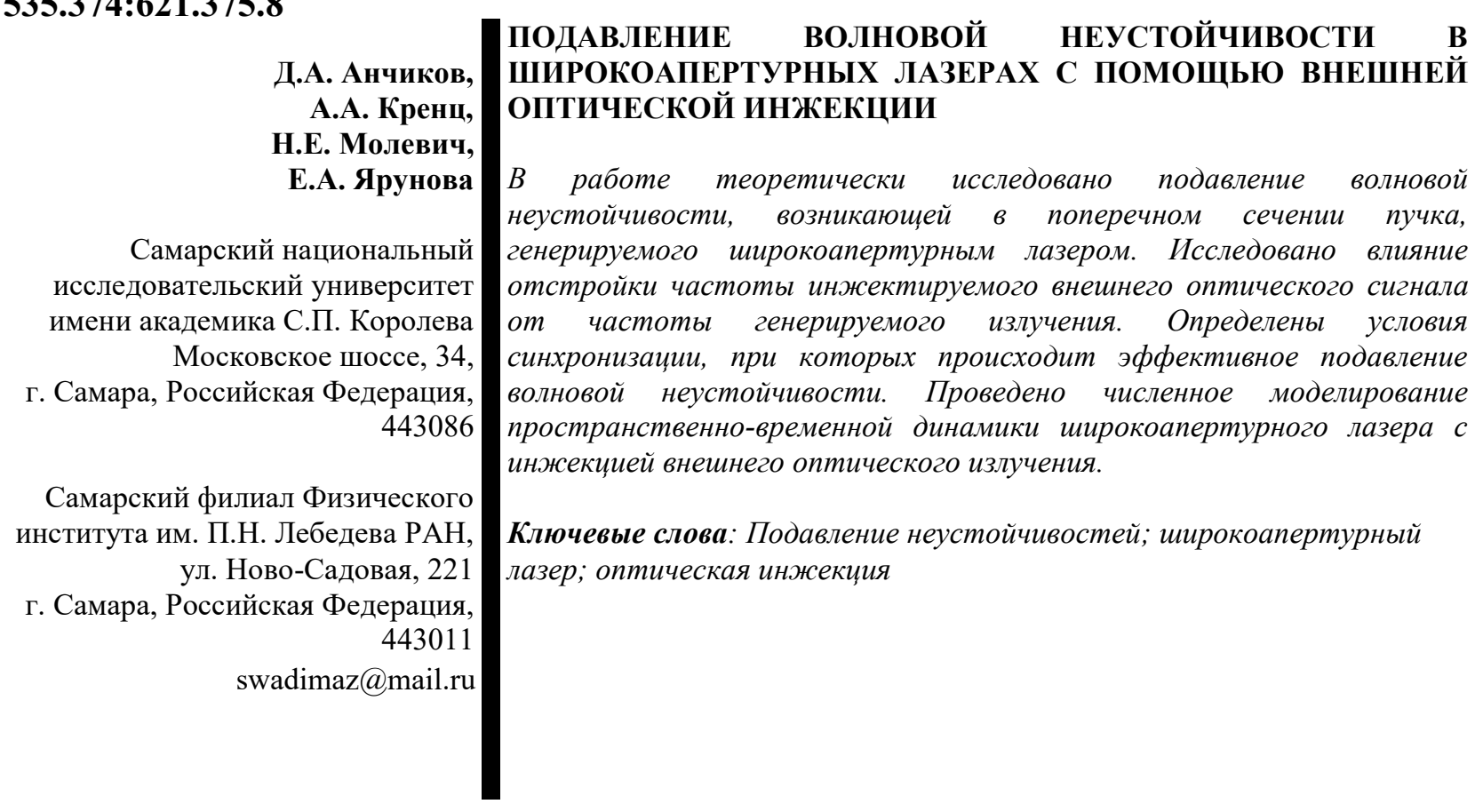

\section{1 Введение}

Разработка ультракомпактных лазерных источников света - одна из наиболее острых и востребованных задач современной оптики, имеющая большое технологическое значение. Такие устройства могут использоваться в различных приложениях, таких как системы обработки данных и оптические каналы связи. Компактные размеры лазера легко позволяют добиться генерации на единственной продольной моде и обеспечивают быстрый отклик лазера благодаря малой длине резонатора. Однако малая длина резонатора приводит к малой мощности выходного излучения, что ограничивает применимость лазера только для систем передачи данных на небольшие расстояния. Наиболее естественным способом обойти это ограничение и увеличить мощность без отказа от всех приемуществ конфигурации с коротким резонатором - использование широкоапертурных лазеров с большими поперечными размерами. Этот подход применим, в частности, для твердотельных микрочиповых лазеров

полупроводниковых лазеров с вертикальным резонатором. Однако увеличение поперечных размеров приводит к ухудшению качества генерируемого излучения из-за возрастающей конкуренции большого числа пространственных поперечных мод. Поперечные неустойчивости в широкоапертурных лазерах отвественны за филаментацию излучения и пространственно-временной хаос в поперечном сечении генерируемого пучка, приводящих к значительному уменьшению пространственной и временной когерентности [1-4]. В большинстве приложений сложная пространственновременная динамика лазера является нежелательным эффектом. Поэтому для практических приложений крайне желательно подавить эти пространственновременные поперечные неустойчивости.

\title{
1 Математическая модель
}

Математическое пространственно-временной ширкоапертурного лазера внешнего оптического моделирование динамики

c инжкцией излучения 
проводилось с помощью системы уравнений Максвелла-Блоха $[5,6]$ :

$$
\left\{\begin{array}{l}
\frac{\partial E}{\partial t}=i a \Delta_{\perp} E+\sigma\left(P-(1-i \delta) E+E_{i n j} e^{i \theta t}\right), \\
\frac{\partial P}{\partial t}=-(1+i \delta) P+D E \\
\frac{\partial D}{\partial t}=-\gamma\left[D-r+\frac{1}{2}\left(E^{*} P+P^{*} E\right)\right],
\end{array}\right.
$$

где $E, P, D$ - безразмерные огибающие электрического поля, поляризации и инверсии населённости соответственно; $\gamma=\gamma_{\|} / \gamma_{\perp}$ и $\sigma=k / \gamma_{\perp}$, где $\gamma_{\perp}, \gamma_{\|}$и $k$ - скорости релаксации поляризации среды, инверсии населённости и электрического поля в резонаторе соответственно; $\delta=\left(\omega_{21}-\omega\right) /\left(\gamma_{\perp}+k\right) \quad$ - обезразмеренная отстройка между центром линии усиления и частотой резонатора; $\quad a=c^{2} /\left(2 \omega \gamma_{\perp} d^{2}\right)$ дифракционный параметр, где $d$ характерный пространственный размер задачи; $r$ - величина накачки, нормированная на её пороговое значение. Внешняя инжекция характеризуется двумя параметрами: $\quad E_{i n j} \quad$ - амплитуда инжектируемого сигнала и $\theta$ - частотная расстройка между инжектируемым сигналом и генерируемым излучением.

\section{2 Стабилизирующее влияние инжекции}

Моделирование динамики проводилось при параметрах, соответствующих широкоапертурным полупроводниковым лазерам: $\sigma=0.025, \gamma=5 \cdot 10^{-5}, a=10^{-4}, r=1.2$. Проведенный анализ устойчивости показал, что существуеют области параметров инжектируемого сигнала, при которых происходит эффективное подавление волновой неустойчивости в широкоапертурно лазере. На рисунке 1 серым цветом показана параметрическая область, в которой происходит эффективное подавление неустойчивых поперечных мод.

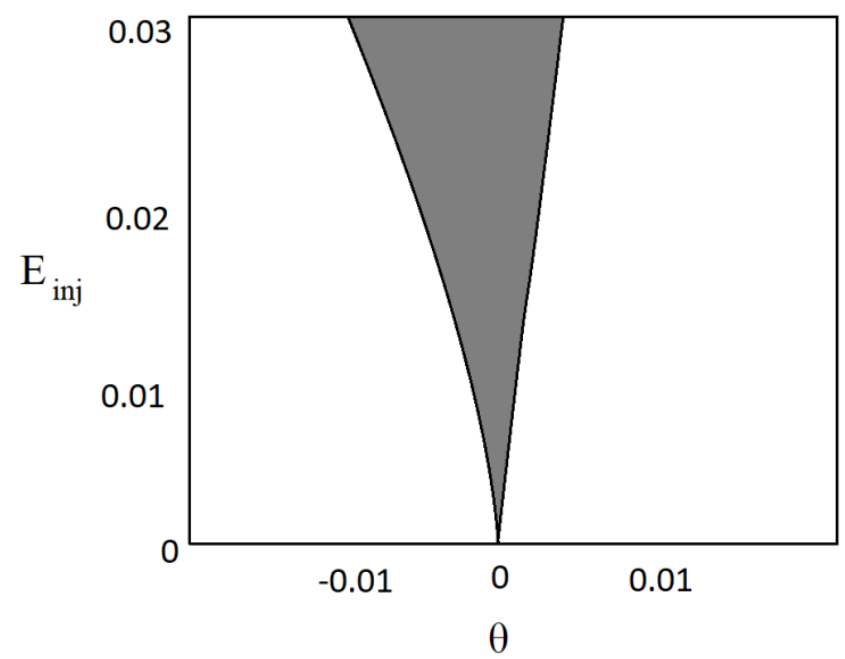

Рисунок 1. Биуркационная диаграмма. Серым цветом показана область параметров инжектируемого сигнала, при которой происходит эффективное подавление неустойчивых поперечных мод

Аналитические результаты были подтверждены прямым численным моделирование системы лазерных уравнений (1).

\section{3 Заключение}

В представленной работе проведён анализ устойчивости для приосевой стационарной генерации в широкоапертурных лазерах. Аналитически показано, что инжекция внешнего излучения подавляет волновую неустойчивость, присущуую широкоапертурным лазерам, даже при учете частотной расстройки между инжектируемым сигналом и генерируемым излучением. Определены параметры инжектируемого сигнала, при которых происходит эфективное подавление неустойчивых пространственных мод. Численное моделирование показало, что предложенный подход позволяет эффективно подавлять неустойчивые поперечные моды и улучшает характеристики излучения лазера.

\section{4 Благодарности}

Работа выполнена при финансовой поддерже Российского фонда 
фундаментальных исследований грант 1832-00704 мол_а.

\section{Список использованных источников}

[1] Fischer, I. Complex spatio-temporal dynamics in the near-field of a broad-area semiconductor laser / I. Fischer, O. Hess, W. Elsäßer, E. Gobel // Europhysics Letters. - 1996. - 35. - Issue 8. - P. 579-84.

[2] Otsuka K. Generation of vortex array beams from a thin-slice solid-state laser with shaped wideaperture laser-diode pumping / K. Otsuka, S. Chu // Optics Letters. - 2009. - N. 34. - Issue 1. - P. 10-12.

[3] Babushkin I. V. Eigenmodes and symmetry selection mechanisms in circular large-aperture vertical- cavity surface-emitting lasers / I. V. Babushkin, N. A. Loiko, T. Ackermann // Physical Review E. - 2004. - Vol. 69. - Issue 6. - 066205.

[4] Gensty T. Wave chaos in real-world vertical-cavity surface-emitting lasers / T. Gensty, K. Becker, I. Fischer, W. Elsäßer, C. Degen, P. Debernardi, G.P. Bava // Physical Review Letters. - 2005. - Vol. 94. - 233901.

[5] Lugiato L.A. Instabilities and spatial complexity in a laser / L.A. Lugiato, G-L. Oppo, J.R. Tredicce, L.M. Narducci, Pernigo M.A. // Journal of the Optical Society of America B. - 1990. - Vol. 7(6). - P. 1019-1033.

[6] Khanin Ya.I. Fundamentals of laser dynamics / Ya.I. Khanin // Cambridge: International Science Publishing. 2006. 
Dmitriy A. Anchikov, Anton A. Krents, Nonna E. Molevich, Elizaveta A. Yarunova

Samara University 34, Moskovskoe shosse, Samara, Russian Federation 443086

Lebedev Physical Institute 221, Novo-Sadovaya str., Samara, Russian Federation 443011

swadimaz@mail.ru
SUPPRESSION OF WAVE INSTABILITY IN BROAD-AREA LASERS BY THE EXTERNAL OPTICAL INJECTION

We theoretically investigate suppression of travelling wave instability in the transverse section of broad-area lasers. We investigate influence of the detuning between injected signal and generated radiation. The synchronization conditions were obtained under which the effective suppression of the wave instability occurs. Numerical simulation of the spatiotemporal dynamics of a broad-area laser with injection of external optical signal is carried out.

Key words: Suppression of instabilities; broad-area laser; optical injection

\section{References}

[1] Fischer, I., Hess, O., Elsäßer, W. and Gobel, E. (1996), "Complex spatio-temporal dynamics in the near-field of a broad-area semiconductor laser", Europhysics Letters, no. 35, pp. 579-84.

[2] Otsuka, K. and Chu, S. (2009), "Generation of vortex array beams from a thin-slice solid-state laser with shaped wide-aperture laser-diode pumping", Optics Letters, no. 34, issue I, pp. 10-12.

[3] Babushkin, I.V., Loiko, N.A. and Ackermann, T. (2004), "Eigenmodes and symmetry selection mechanisms in circular large-aperture vertical-cavity surface-emitting lasers", Physical Review E, vol. 69, issue 6, 066205.

[4] Gensty, T., Becker, K., Fischer, I., Elsäßer, W., Degen, C., Debernardi, P. and Bava, G.P. (2005), "Wave chaos in real-world vertical-cavity surface-emitting lasers", Physical Review Letters, vol. 94, 233901.

[5] Lugiato, L.A., Oppo, G-L, Tredicce, J.R., Narducci, L.M. and Pernigo, M.A. (1990), "Instabilities and spatial complexity in a laser", Journal of the Optical Society of America B, vol. 7(6), pp. 1019-1033.

[6] Khanin, Ya.I. (2006), "Fundamentals of laser dynamics", Cambridge: International Science Publishing. 